\title{
Considering Frailty in SARS-CoV-2 Vaccine Development: How Geriatricians Can Assist
}

\author{
Melissa K Andrew (iD) ${ }^{1,2}$ \\ Kenneth E Schmader ${ }^{3}$ \\ Kenneth Rockwood (1D) \\ Barry Clarke ${ }^{4}$ \\ Janet E McElhaney ${ }^{5}$ \\ 'Department of Medicine, Division of \\ Geriatric Medicine, Dalhousie University, \\ Halifax, Nova Scotia, Canada; ${ }^{2}$ Canadian \\ Center for Vaccinology, Halifax, Nova \\ Scotia, Canada; ${ }^{3}$ Division of Geriatrics, \\ Duke University Medical Center and \\ GRECC, Durham Veterans Affairs \\ Medical Center, Durham, North \\ Carolina, USA; ${ }^{4}$ Department of Family \\ Medicine, Dalhousie University, Halifax, \\ Canada; ${ }^{5}$ Health Sciences North \\ Research Institute, Sudbury, Ontario, \\ Canada
}

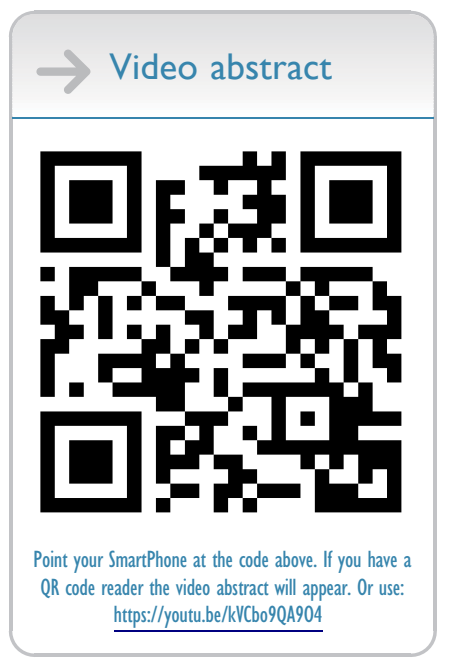

Correspondence: Melissa K Andrew Department of Medicine, Division of Geriatric Medicine, Dalhousie University, Veterans Memorial Building, 5955 Veterans Memorial Lane, Halifax, NS, B3H 2EI, Canada

Tel +l $902473-4995$

Email mandrew@dal.ca

\begin{abstract}
The COVID-19 pandemic has disproportionately impacted frail older adults, especially residents of long-term care (LTC) facilities. This has appropriately led to prioritization of frail older adults and LTC residents, and those who care for them, in the vaccination effort against COVID-19. Older adults have distinct immunological, clinical, and practical complexity, which can be understood through a lens of frailty. Even so, frailty has not been considered in studies of COVID-19 vaccines to date, leading to concerns that the vaccines have not been optimally tailored for and evaluated in this population even as vaccination programs are being implemented. This is an example of how vaccines are often not tested in Phase 1/2/3 clinical trials in the people most in need of protection. We argue that geriatricians, as frailty specialists, have much to contribute to the development, testing and implementation of COVID-19 vaccines in older adults. We discuss roles for geriatricians in ten stages of the vaccine development process, covering vaccine design, trial design, trial recruitment, establishment and interpretation of illness definitions, safety monitoring, consideration of relevant health measures such as frailty and function, analysis methods to account for frailty and differential vulnerability, contributions in regulatory and advisory roles, post-marketing surveillance, and program implementation and public health messaging. In presenting key recommendations pertinent to each stage, we hope to contribute to a dialogue on how to push the field of vaccinology to embrace the complexity of frailty. Making vaccines that can benefit frail older adults will benefit everyone in the fight against COVID-19.
\end{abstract}

Keywords: SARS-CoV-2, COVID-19, vaccine, frailty, frail elderly, geriatrics

As the COVID-19 pandemic has evolved, it has become clear that outcomes are highly age-dependent. Mortality increases exponentially with age after 50 , and most fatalities have occurred in those over age $80{ }^{1,2}$ Even so, outcomes have varied across the full spectrum of asymptomatic to catastrophic illness in every age group including the oldest old. This variability in health status and outcomes can be understood through a lens of frailty, which is a state of increased vulnerability to adverse health outcomes. ${ }^{3}$ Frailty tends to increase with age but goes beyond simple time-based measures to understand the variability in health status and vulnerability among people of the same chronological age. ${ }^{4}$

Frailty is increasingly a focus of the public, health care professionals, decision makers and regulators. All want to know how well a given intervention, treatment or vaccine is likely to work across the spectrum of frailty. In the case of vaccination against COVID-19, there is particular interest in understanding how to protect those most at risk, which importantly includes the oldest and frailest adults, and notably 
those who reside in long-term care facilities (LTCFs). Because of this, in many jurisdictions, frail LTCF residents (and those who care for them) are priority groups for vaccination..$^{5-7}$

Prioritizing frail older adults for vaccination stands in opposition to the observation that, as with most clinical trials, to date, COVID vaccine trials have generally excluded frail older adults and residents of LTCF. $^{8,9}$ This knowledge gap between what we need to know and what the trials are telling us could be addressed by conducting appropriate studies in this population. That prospect, though desirable, is in no way guaranteed. We argue that intentional thought about frailty would be beneficial across all stages in the processes of vaccine development, testing and implementation. A starting point is the observation that trial author groups generally have not included geriatricians, despite otherwise representing (appropriately) large study teams with diverse expertise. This is perhaps not surprising due to the historical observation that the field of vaccinology has long centred around pediatrics and public health; given the focus on vaccines for infants and children as undeniably critical aspects of preventive care, the field of pediatric vaccinology is much more developed than the more emerging one of geriatric vaccinology.
Why is this important? Older adults are not simply adults who have been alive for more years. There is an interesting distinction to be made between age and aging. Whereas age implies the simple passage of chronological time, aging brings the added meaning of increased risk of failure over time. If we see the human body as a complex system, an aging system will have increasing risk of failure over time (just as would be the case for a complex mechanical system such as a car). One way to understand this is through the accumulation of deficits, which contributes to this increased risk of failure - this accumulation of deficits has the added benefit of being quantifiable, as is done in the frailty index.

Geriatricians are frailty specialists. In the context of this paper's discussion and recommendations, a geriatrician is a clinician and/or academic with special training and expertise in the care of frail older adults and in understanding principles of aging in relation to vaccines. As vaccines are developed and targeted to older adults, there are many important roles for geriatricians across all stages in vaccine development, testing and implementation (Figure 1).

\section{Vaccine Design}

Immune responses tend to wane and become dysregulated with age, through processes known as immunosenescence. Notably, immunosenescence does not occur uniformly

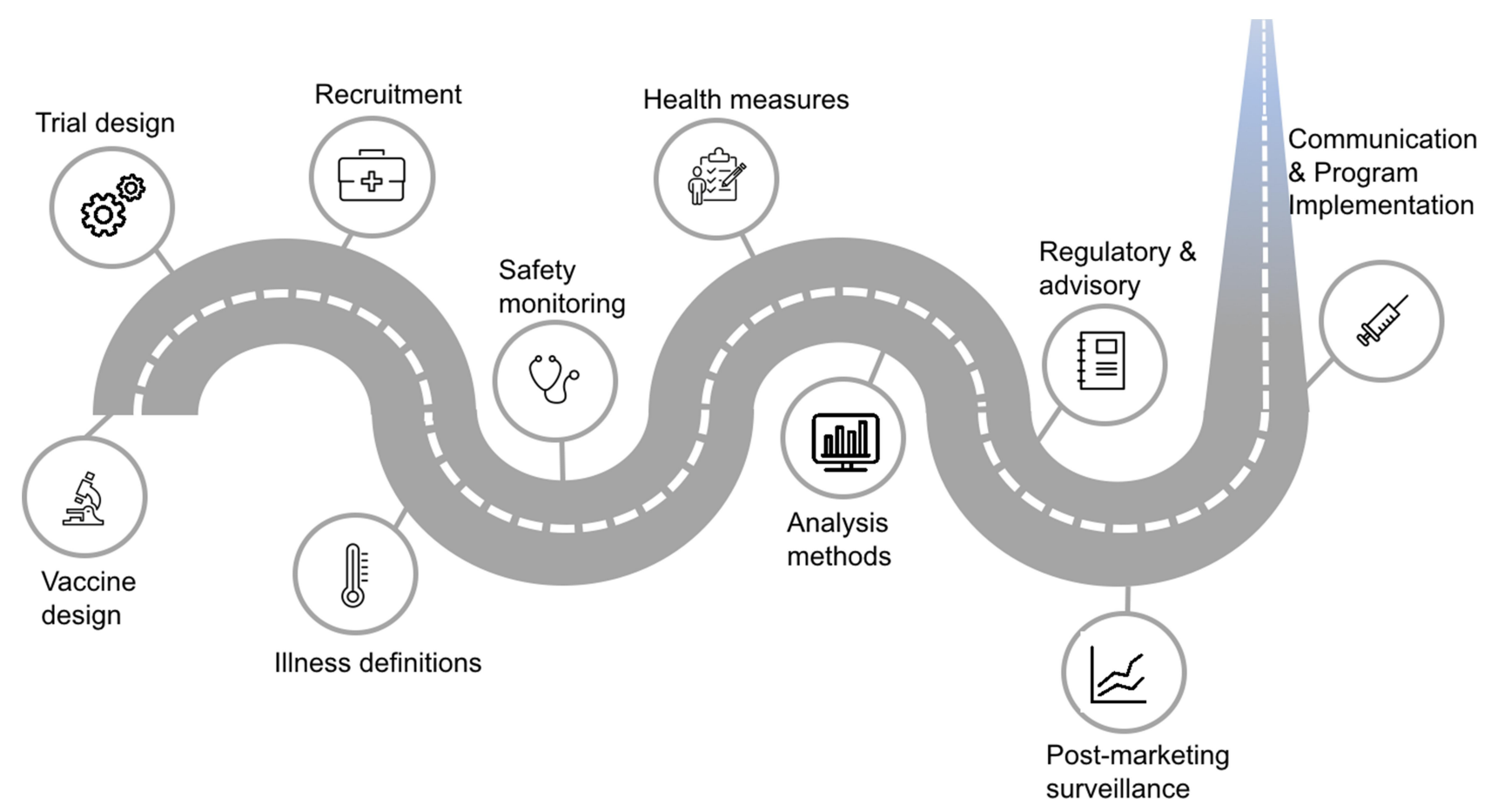

Figure I Roles for geriatricians arise across all stages in vaccine development, testing and implementation. 
across all older adults as they age. Indeed, this variability is hypothesized to be a contributor to frailty itself. ${ }^{10,11}$

It is thus important to design vaccines to overcome and stimulate immune responses that may be dysregulated and immunosenescent. Advances in nucleic acid, vector and adjuvanted vaccine platforms offer rich opportunities in this area. Newer adjuvants may overcome age-related defects in innate immunity including frail individuals. ${ }^{12,13}$ Cell-mediated immunity (CMI) is particularly important in the setting of frailty and immunosenescence so vaccines should be designed to optimize CMI responses. $^{11,14}$ Along with this, vaccine immunogenicity trials need to include measures of CMI that better reflect the immune responses of frail older adults. A small but growing community of geriatric (and geriatrician) immunologists bring an important perspective to this basic science work. For example, the decline in CMI responses to influenza vaccination appears to be a limitation of current influenza vaccines. $^{15}$ In contrast, COVID-19 RNA vaccines stimulate a robust CMI response that is associated with the preserved $95 \%$ protection rates against COVID-19 observed in older adults in the Phase III trials. $^{16}$

Key recommendation: COVID-19 vaccine design should account for immunosenescence and the importance of cell-mediated immune responses in frail older adults.

\section{Trial Design}

Clinical trials are designed with the intent of isolating the effect of intervention being studied; in order to achieve this, recruitment generally focuses on healthy (and more often young) volunteers, and participants with underlying health complexity are often excluded. This age discrimination can be direct (eg, arbitrary age cutoffs) or indirect (eg, exclusions on the basis of comorbidities, disability, medication use, or failing to adapt procedures and materials for sensory impairments). ${ }^{17}$ Excluding older and frailer individuals may be quite appropriate in early phase (I-II) studies, where basic questions about safety and dose finding are being asked. However, in later phases (II-III) when questions turn to optimizing immune responses, efficacy and safety in the target populations, excluding older and frail participants is problematic. If vaccines are not evaluated in populations most at risk, questions about their efficacy, safety, immunogenicity and reactogenicity will remain unanswered until they are deployed in these realworld populations in the rollout of vaccination programs. Innovative clinical trial designs, such as adaptive, cluster or pragmatic clinical trials, offer the opportunity to better study frail individuals. ${ }^{18}$ Relevant to the challenge of immunosenescence, evidence from other vaccines suggests that frail older adults may benefit from higher doses of antigen (eg, high-dose influenza vaccine $)^{19-21}$ or inclusion of adjuvants to stimulate immune responses (eg, adjuvanted influenza vaccine, recombinant shingles vaccine). ${ }^{13,18,22,23}$ If we do not include older frail participants in vaccine studies, this opportunity to optimize the products for their needs will be missed.

Key recommendation: Particularly in later phases, vaccine trials should be designed to include (and not explicitly exclude) frail older people.

\section{Trial Recruitment}

Beyond defining appropriate inclusion and exclusion criteria, recruitment can be a particular challenge for frail older adults. Practical factors should be considered: geography, transportation, messaging, access to recruitment and clinical sites, and facilitating the consent processes for people with sensory and/or cognitive impairment. Geriatricians can help overcome barriers to recruitment in frail older adults including cognitive impairment, dementia, visual impairment, hearing impairment, dysmobility, consideration of goals of care, and caregiver concerns. These issues present important challenges at any time but have presented unique barriers during COVID times. For example, recruiting frail participants for inperson visits during a lockdown or "shelter in place" advisory in the surrounding community ${ }^{24,25}$ and conducting virtual information and consent discussions where technology (eg, tablet devices) may be unfamiliar or present barriers due to sensory impairments are all very real considerations. ${ }^{26}$ Even so, when means of working around these barriers are developed, the payoff will be much better population representativeness. Overcoming these sorts of barriers is part of the routine skill set of geriatricians in clinical settings (even pre-COVID). Considering the setting of recruitment is essential. For example, study recruitment within long-term care facilities could help facilitate safe, pragmatic and respectful access to relevant potential participant populations. As with any marginalized population, relationships of trust are key to inclusion and recruitment. A trusted clinician and/or LTCF medical director who is embedded in a potential study setting is well positioned to work from this place of trust and open communication to help reach potential participants. 
Geriatricians are likely to be such people or to know these networks well.

Key recommendation: Recruitment of frail older adults will be optimized when we consider how to best reach targeted participants and start with a relationship of trust.

\section{Establish and Interpret IIIness Definitions}

As is foundational to geriatric medicine, but perhaps less well known outside it, older adults tend to present differently with acute illness compared with younger adults; this is particularly the case when these older adults are frail and/or who live with dementia. These "atypical presentations" of illness, such as delirium, functional decline and reductions in mobility, are actually more the norm than the exception when frail older adults are ill. ${ }^{27}$ This can be perplexing until we think back to the complex system analogy. The highest order functions of our human complex system include complex cognition and divided attention, opposable thumbs and the use of tools, and upright bipedal ambulation. ${ }^{28}$ When the vulnerable complex system of the frail older adult is under stress, these may be some of the first things to go. ${ }^{29}$ When these high-order functions fail, we see acute confusion (delirium), functional decline and falls. ${ }^{28}$ The frailer an older adult is, the more prone to these "atypical" presentations they will be.

In COVID-19 vaccine trials, illness case definitions are critical because they are the mechanism through which potential cases of infection are identified; importantly, this is the foundation upon which vaccine efficacy (VE) calculations are based. ${ }^{30-32}$ If cases are missed due to not meeting inappropriately rigid case definitions, the trial will not result in an accurate measure of VE. In the case of COVID-19, a frail older adult may thus present with symptoms that are outside the usual testing criteria in any clinical setting or clinical trial. ${ }^{33,34}$ In addition to tending to presenting atypically, older adults living with underlying cognitive impairment or dementia may have difficulty recognizing or communicating their symptoms. ${ }^{35}$ A geriatrician will be well positioned to help design trial case definitions to pick up on these atypical presentations.

Key recommendation: Atypical presentations of illness (more common in frail older adults) should be considered in COVID-19 vaccine trial case definitions, which are the foundation of vaccine efficacy calculations.

\section{Safety Monitoring}

Including geriatricians on Data and Safety Monitoring Boards (DSMB) would be important for trials conducted in frail older adults. Just as it would be unthinkable to do a study in children with no pediatrician expertise in evaluating potential adverse events, the same should apply for the geriatric population. This is particularly important given the arguments presented above that older adults are not simply adults who are older and illness presentations are often unique to this population. Also, frail older adults are a group which also has a high level of background events. Including geriatricians in the trial study team will add useful expertise in interpreting the numerous adverse events that occur following the intervention. Adverse event rates are much higher in older adults, especially in frail older adults than younger individuals. The volume and types of events need careful evaluation by clinicians who understand this population. All of these factors present challenges to interpreting potential safety signals and to defining and implementing appropriate pause rules, and geriatricians stand to contribute important expertise.

Key recommendation: When a study includes frail older adult participants, a geriatrician should be on the DSMB.

\section{Health Measures Used in Vaccine Trials}

Geriatricians are also well positioned to assist with the measures of health (both baseline and outcomes) that are used in clinical trials. It is feasible to include validated health outcome measures relevant to older adults in vaccine clinical trials. ${ }^{36,37}$ As a starting place, it is important to include measures of frailty; otherwise, it is not possible to evaluate outcomes by level of frailty, generate VE estimates across frailty categories or adjust VE estimates for frailty, which has been shown to be both highly informative and feasible in studies of influenza and shingles. ${ }^{13,38}$ This is becoming increasingly important for regulators as well, so consideration of frailty in vaccine trials will set the stage for this aspect of regulatory approval. Outcomes measured in clinical trials are often not the ones that are valued and relevant to older adults. This is the case even for mortality; despite being seen as the worst-case scenario in most trials, many older adults themselves value outcomes such as functional independence and quality of life over quantity of life, so including these measures helps to more clearly measure (and allow 
communication of) benefits in these domains. ${ }^{39,40}$ This, in turn, can help inform public health messaging - for example in the case of influenza, advocacy groups representing older adults develop messages centered around AVOIDing frailty (including through vaccination) ${ }^{41}$ and preventing persistent functional disability following recovery from an acute infection by promoting adult vaccination. ${ }^{42}$ Rapid assessment methods for evaluating function are available and may be well suited to this purpose. ${ }^{43,44}$

Key recommendation: Measures of health and outcomes (eg, frailty and function) that are relevant to frail older adults should be included in COVID-19 vaccine trials.

\section{Analysis Methods to Account for Frailty and Differential Vulnerability}

Even when frailty has not been included up front in a complex trial design, it may be possible to retrospectively generate a frailty measure using data that have already been collected. In the case of clinical trials where baseline health and symptom data have been collected, it is possible to create a deficit accumulation frailty index measure. This has been demonstrated, for example, in the shingles vaccine literature $e^{13,45}$ and in cardiovascular clinical trials. ${ }^{46-48}$ This allows determination of the spectrum of frailty represented among the trial participants as well as estimates of stratified VE by level of frailty. ${ }^{13}$ In other cases (such as observational studies) where individuallevel health data are less well characterized, propensity scores and other complex statistical methods may be applied to generate estimates of differential vulnerability. ${ }^{49-52}$ In either case, geriatricians with expertise in frailty measurement would be well positioned to contribute to the study team.

Key recommendation: Even when a measure of frailty has not been included up front in a clinical trial of COVID-19 vaccines, efforts should be made to account for frailty in statistical analyses (eg, use of a retrospectively generated frailty index or propensity score with stratified estimates of vaccine efficacy, immunogenicity, reactogenicity and safety).

\section{Regulatory and Advisory Contributions}

Given the recognition that frail older adults are a key target population for COVID vaccination, it would be reasonable to expect that geriatricians may be involved in informing regulatory decisions and advisory body recommendations. The Centers for Disease Control Advisory Committee on Immunization Practices COVID19 vaccines work group includes a geriatrician representative of the American Geriatrics Society, ${ }^{6}$ and advocating for increased contributions to these activities in other jurisdictions is a reasonable goal.

Key recommendation: When regulatory and advisory decisions are being made concerning vaccines targeting frail older adults, geriatrics expertise should be included.

\section{Post-Marketing Surveillance}

Just as study design is critical in early phases of vaccine testing, once vaccines are in general use, Phase IV and other observational studies benefit from similar considerations of how to best define study populations, measure and account for frailty, and consider relevant geriatric outcomes. Again, geriatricians are well positioned to contribute this expertise. For example, these have been important considerations in influenza burden of disease and VE surveillance, with frailty and function being key embedded measures in the Canadian Immunization Research Network's Serious Outcomes Surveillance Network. Over a decade of active hospital-based surveillance for influenza, this geriatric focus has allowed consideration of the importance of frailty for influenza outcomes and VE and also exploration of the true burden of influenza in older adults by tracking relevant outcomes following the acute illness episode itself, such as persistent declines in function and frailty. ${ }^{38,53,54}$

Key recommendation: Post-implementation surveillance and evaluation of COVID-19 vaccines should incorporate relevant health and outcome measures, such as frailty and function.

\section{Program Implementation and Communication}

Clear and consistent communication is essential for successful vaccine program implementation. This includes communication relating to existing knowledge and evidence gaps pertinent to older age and frailty and addressing special concerns that arise in this population. Examples include management of comfort during vaccination, potential for reactogenicity in already frail vaccine recipients (although evidence from COVID-19 and other vaccines suggests that reactogenicity tends to be lower with increasing age and frailty), ${ }^{13,30-32,55}$ 
and concerns about serious adverse events occurring in the setting of a population with high background events. ${ }^{56}$ This age- and frailty-specific communication is important to help inform and guide immunization practice across settings; integrating this messaging within primary care is particularly essential, and geriatricians are likely to have existing collaborative relationships with primary care and long-term care clinicians. Again, trust is important - although vaccine hesitancy is generally lower among older adults, it is present in the older age groups and also among substitute decision makers (SDMs) and LTCF staff who engage with older persons with cognitive impairment, impacting decision-making capacity to make vaccination decisions. ${ }^{57,58}$

It is also important to have open lines of communication with frailty stakeholders, such as non-governmental organizations and advocacy groups. ${ }^{41,42,59-61}$ Existing relationships, which geriatricians may already have established, are helpful.

Key recommendation: Communication of age- and frailty-specific knowledge and evidence gaps is important to maintain trust in public health messaging about COVID-19 vaccination.

Frail older adults, especially those who live in longterm care facilities, have been among the most impacted to date by the COVID-19 pandemic. They are thus a vulnerable population who have been appropriately prioritized for COVID-19 vaccination. Even so, many knowledge gaps remain relating to the use of COVID-19 vaccination in this population. Given older adults' distinct immunological, clinical, and practical complexity, there are important roles for geriatricians at every step from COVID-19 vaccine development to testing and program implementation. It is our hope that the key recommendations presented here (summarized in Box 1) will stimulate discussion and action in ongoing efforts to use vaccination as a critical element in the battle against COVID-19.

\section{Acknowledgments}

KES received support from NIA P30AG028716. MKA, JEM and KR are part of the Canadian Consortium on Neurodegeneration in Aging (CCNA) Team 14, which investigates how multi-morbidity and frailty modify risk of dementia and patterns of disease expression. The CCNA receives funding from the Canadian Institutes of Health Research (CNA-137794) and partner organizations (www.ccna-ccnv.ca).
Box I Summary of 10 Key Recommendations for Involving Geriatrics Expertise in COVID-19 Vaccine Development, Evaluation and Program Implementation

I. COVID-19 vaccine design should account for immunosenescence and the importance of cell-mediated immune responses in frail older adults.

2. Particularly in later phases, vaccine trials should be designed to include (and not explicitly exclude) frail older people.

3. Recruitment of frail older adults will be optimized when we consider how to best reach targeted participants and start with a relationship of trust.

4. Atypical presentations of illness (more common in frail older adults) should be considered in COVID-19 vaccine trial case definitions, which are the foundation of vaccine efficacy calculations.

5. When a study includes frail older adult participants, a geriatrician should be on the DSMB.

6. Measures of health and outcomes (eg, frailty and function) that are relevant to frail older adults should be included in COVID-19 vaccine trials.

7. Even when a measure of frailty has not been included up front in a clinical trial of COVID-19 vaccines, efforts should be made to account for frailty in statistical analyses (eg, use of a retrospectively generated frailty index or propensity score with stratified estimates of vaccine efficacy, immunogenicity, reactogenicity and safety).

8. When regulatory and advisory decisions are being made concerning vaccines targeting frail older adults, geriatrics expertise should be included.

9. Post-implementation surveillance and evaluation of COVID-19 vaccines should incorporate relevant health and outcome measures, such as frailty and function.

10. Communication of age- and frailty-specific knowledge and evidence gaps is important to maintain trust in public health messaging about COVID-19 vaccination.

\section{Disclosure}

MKA reports grants from Sanofi, GSK, Pfizer, Canadian Frailty Network and the Public Health Agency of Canada for research on frailty in relation to vaccine preventable illness and outcomes, and honoraria from Sanofi, Pfizer and Seqirus unrelated to the present work. KES is a member of the Centers for Disease Control Advisory Committee on Immunization Practices COVID-19 vaccines work group. KR reports that he founded (as DGI Clinical) and is the Chief Science Officer of Ardea Outcomes, which has several contracts with pharma and medical device manufacturers. BC reports no conflicts. JEM reports personal fees from GSK Merck, Medicago, RestorBio, and Sanofi and travel support for scientific presentations from Sanofi and GSK, outside the submitted work; and payments to her institution from GlaxoSmithKline, Merck, Sanofi, Pfizer, Medicago, and RestorBio outside of the 
submitted work. The authors reported no other potential conflicts of interest for this work.

\section{References}

1. Bonanad C, Garcia-Blas S, Tarazona-Santabalbina F, et al. The effect of age on mortality in patients with COVID-19: a meta-analysis with 611,583 subjects. J Am Med Dir Assoc. 2020;21(7):915-918. doi:10.1016/j.jamda.2020.05.045

2. Kang SJ, Jung SI. Age-related morbidity and mortality among patients with COVID-19. Infect Chemother. 2020;52(2):154-164. doi:10.3947/ic.2020.52.2.154

3. Clegg A, Young J, Iliffe S, Rikkert MO, Rockwood K. Frailty in elderly people. Lancet. 2013;381(9868):752-762. doi:10.1016/ S0140-6736(12)62167-9

4. Rockwood K, Howlett SE. Age-related deficit accumulation and the diseases of ageing. Mech Ageing Dev. 2019;180:107-116. doi:10.1016/j.mad.2019.04.005

5. Joint Committee on Vaccination and Immunisation. Priority groups for coronavirus (COVID-19) vaccination: advice from the JCVI; 2020. Available from: https://www.gov.uk/government/publications/ priority-groups-for-coronavirus-covid-19-vaccination-advice-fromthe-jcvi-30-december-2020. Accessed February 12, 2021.

6. Dooling K, Marin M, Wallace M, et al. The advisory committee on immunization practices' updated interim recommendation for allocation of COVID-19 Vaccine - United States, December 2020. MMWR Morb Mortal Wkly Rep. 2021;69(5152):1657-1660. doi:10.15585/ mmwr.mm695152e2

7. National Advisory Committee on Immunization. Preliminary guidance on key populations for early COVID-10 immunization; 2020. Available from: https://www.canada.ca/en/public-health/services/ immunization/national-advisory-committee-on-immunization-naci /guidance-key-populations-early-covid-19-immunization.html. Accessed February 12, 2021.

8. Andrew MK, McElhaney JE. Age and frailty in COVID-19 vaccine development. Lancet. 2021;396(10267):1942-1944. doi:10.1016/ S0140-6736(20)32481-8

9. McElhaney JE, Taddio A, Harmon SHE. The elderly, the frail, and COVID-19 vaccines: what we know so far; 2021. Available from: https://rsc-src.ca/en/voices/elderly-frail-and-covid-19-vaccines-whatwe-know-so-far. Accessed April 9, 2021. Royal Society of Canada.

10. Fulop T, McElhaney J, Pawelec G, et al. Frailty, inflammation and immunosenescence. Interdiscip Top Gerontol Geriatr. 2015;41:26-40.

11. McElhaney JE, Verschoor CP, Andrew MK, Haynes L, Kuchel GA, Pawelec G. The immune response to influenza in older humans: beyond immune senescence. Immun Ageing. 2020;17:10 doi:10.1186/s12979-020-00181-1

12. Cunningham AL, Lal H, Kovac M, et al. Efficacy of the herpes zoster subunit vaccine in adults 70 years of age or older. $N$ Engl $J$ Med. 2016;375(11):1019-1032. doi:10.1056/NEJMoa1603800

13. Curran D, Kim JH, Matthews S, et al. Recombinant zoster vaccine is efficacious and safe in frail individuals. $J$ Am Geriatr Soc. 2020;69:744-752. doi:10.1111/jgs.16917

14. McElhaney JE, Andrew MK, Haynes L, Kuchel GA, McNeil SA, Pawelec G. Influenza vaccination: accelerating the process for new vaccine development in older adults. Interdiscip Top Gerontol Geriatr. 2020;43:98-112.

15. McElhaney JE, Ewen C, Zhou X, et al. Granzyme B: correlates with protection and enhanced CTL response to influenza vaccination in older adults. Vaccine. 2009;27(18):2418-2425. doi:10.1016/j. vaccine. 2009.01.136

16. Sahin U, Muik A, Vogler I, et al. BNT162b2 induces SARS-CoV2-neutralising antibodies and T cells in humans; 2021. Available from: https://www.medrxiv.org/content/10.1101/2020.12.09.20245175v1. Accessed April 9, 2021. medRxiv preprint server; preprint.
17. Crome P. Clinical trials in older people: are we being ageist? Clin Invest. 2012;2(3):231-234. doi:10.4155/cli.12.2

18. McConeghy KW, Davidson HE, Canaday DH, et al. Clusterrandomized trial of adjuvanted vs. non-adjuvanted trivalent influenza vaccine in 823 U.S. nursing homes. Clin Infect Dis. 2020. doi:10.1093/cid/ciaa1233

19. Gravenstein S, Davidson HE, Taljaard M, et al. Comparative effectiveness of high-dose versus standard-dose influenza vaccination on numbers of US nursing home residents admitted to hospital: a cluster-randomised trial. Lancet Respir Med. 2017;5(9):738-746. doi:10.1016/S2213-2600(17)30235-7

20. DiazGranados CA, Dunning AJ, Kimmel M, et al. Efficacy of high-dose versus standard-dose influenza vaccine in older adults. N Engl J Med. 2014;371(7):635-645. doi:10.1056/NEJMoa1315727

21. DiazGranados CA, Dunning AJ, Robertson CA, Talbot HK, Landolfi V, Greenberg DP. Efficacy and immunogenicity of high-dose influenza vaccine in older adults by age, comorbidities, and frailty. Vaccine. 2015;33(36):4565-4571. doi:10.1016/j.vaccine.2015.07.003

22. Banzhoff A, Nacci P, Podda A. A new MF59-adjuvanted influenza vaccine enhances the immune response in the elderly with chronic diseases: results from an immunogenicity meta-analysis. Gerontology. 2003;49(3):177-184. doi:10.1159/000069172

23. Andrew MK, Bowles SK, Pawelec G, et al. Influenza vaccination in older adults: recent innovations and practical applications. Drugs Aging. 2019;36(1):29-37. doi:10.1007/s40266-018-0 $597-4$

24. Gotz FM, Gvirtz A, Galinsky AD, Jachimowicz JM. How personality and policy predict pandemic behavior: understanding sheltering-inplace in 55 countries at the onset of COVID-19. Am Psychol. 2021;76 (1):39-49. doi:10.1037/amp0000740

25. Bailey L, Ward M, DiCosimo A, et al. Physical and mental health of older people while cocooning during the COVID-19 Pandemic. QJM. 2021. doi:10.1093/qjmed/hcab015

26. Monaco A, Palmer K, Holm Ravn Faber N, et al. Digital health tools for managing noncommunicable diseases during and after the COVID-19 pandemic: perspectives of patients and caregivers. $J$ Med Internet Res. 2021;23(1):e25652. doi:10.2196/25652

27. Jarrett PG, Rockwood K, Carver D, Stolee P, Cosway S. Illness presentation in elderly patients. Arch Intern Med. 1995;155 (10):1060-1064. doi:10.1001/archinte.1995.00430100086010

28. Rockwood K, Theou O. Using the clinical frailty scale in allocating scarce health care resources. Can Geriatr J. 2020;23(3):210-215. doi:10.5770/cgj.23.463

29. Hadley EC, Kuchel GA, Newman AB, Workshop S. Participants. Report: NIA workshop on measures of physiologic resiliencies in human aging. J Gerontol a Biol Sci Med Sci. 2017;72(7):980-990. doi:10.1093/gerona/glx015

30. Voysey M, Clemens SAC, Madhi SA, et al. Safety and efficacy of the ChAdOx1 nCoV-19 vaccine (AZD1222) against SARS-CoV-2: an interim analysis of four randomised controlled trials in Brazil, South Africa, and the UK. Lancet. 2021;397(10269):99-111.

31. Baden LR, El Sahly HM, Essink B, et al. Efficacy and Safety of the mRNA-1273 SARS-CoV-2 Vaccine. $N$ Engl J Med. 2020.

32. Polack FP, Thomas SJ, Kitchin N, et al. Safety and Efficacy of the BNT162b2 mRNA Covid-19 Vaccine. N Engl J Med. 2020;383 (27):2603-2615. doi:10.1056/NEJMoa2034577

33. Rawle MJ, Bertfield DL, Brill SE. Atypical presentations of COVID-19 in care home residents presenting to secondary care: a UK single centre study. Aging Med (Milton). 2020;3(4):237-244. doi:10.1002/agm2.12126

34. Gan JM, Kho J, Akhunbay-Fudge M, et al. Atypical presentation of COVID-19 in hospitalised older adults. Ir J Med Sci. 2020. doi:10.1007/s11845-020-02372-7

35. Palermo S. Covid-19 pandemic: maximizing future vaccination treatments considering aging and frailty. Front Med (Lausanne). 2020;7:558835. doi:10.3389/fmed.2020.558835 
36. Schmader KE, Johnson GR, Saddier P, et al. Effect of a zoster vaccine on herpes zoster-related interference with functional status and health-related quality-of-life measures in older adults. $\mathrm{J} \mathrm{Am}$ Geriatr Soc. 2010;58(9):1634-1641. doi:10.1111/j.15325415.2010.03021.x

37. Schmader KE, Levin MJ, Grupping K, et al. The impact of reactogenicity after the first dose of recombinant zoster vaccine on the physical functioning and quality of life of older adults: an open-label, Phase III trial. J Gerontol a Biol Sci Med Sci. 2019;74 (8):1217-1224. doi:10.1093/gerona/gly218

38. Andrew MK, Shinde V, Ye L, et al. The importance of frailty in the assessment of influenza vaccine effectiveness against influenza-related hospitalization in elderly people. J Infect Dis. 2017;216(4):405-414. doi:10.1093/infdis/jix282

39. Disabled Living Foundation. Losing independence is a bigger ageing worry than dying; 2009. Available from: http://www.dlf.org.uk/blog/ losing-independence-bigger-ageing-worry-dying. Accessed April 9, 2021.

40. Phelan EA, Anderson LA, LaCroix AZ, Larson EB. Older adults' views of "successful aging" - how do they compare with researchers' definitions? J Am Geriatr Soc. 2004;52(2):211-216. doi:10.1111/ j.1532-5415.2004.52056.x

41. Canadian Frailty Network. AVOID Frailty; 2019. Available from: https://www.cfn-nce.ca/frailty-matters/avoid-frailty/. Accessed February 12, 2021.

42. International Federation on Ageing. Vaccines4Life; 2021. Available from: https://www.vaccines4life.com. Accessed February 12, 2021.

43. McGarrigle L, Squires E, Wallace LMK, et al. Investigating the feasibility and reliability of the Pictorial Fit-Frail Scale. Age Ageing. 2019;48(6):832-837. doi:10.1093/ageing/afz111

44. Theou O, Andrew M, Ahip SS, et al. The pictorial fit-frail scale: developing a visual scale to assess frailty. Can Geriatr J. 2019;22 (2):64-74. doi:10.5770/cgj.22.357

45. Curran D, Andrew MK, Levin MJ, et al. Evaluation of two frailty indices, with practical application in a vaccine clinical trial. Hum Vaccin Immunother. 2019;15(12):2960-2968. doi:10.1080/ 21645515.2019.1622974

46. Wilkinson C, Wu J, Searle SD, et al. Clinical outcomes in patients with atrial fibrillation and frailty: insights from the ENGAGE AF-TIMI 48 trial. BMC Med. 2020;18(1):401. doi:10.1186/s12916020-01870-w

47. Dewan P, Jackson A, Jhund PS, et al. The prevalence and importance of frailty in heart failure with reduced ejection fraction - an analysis of PARADIGM-HF and ATMOSPHERE. Eur J Heart Fail. 2020;22 (11):2123-2133. doi:10.1002/ejhf.1832

48. Farooqi MAM, Gerstein H, Yusuf S, Leong DP. Accumulation of deficits as a key risk factor for cardiovascular morbidity and mortality: a pooled analysis of 154000 individuals. J Am Heart Assoc. 2020;9(3):e014686. doi:10.1161/JAHA.119.014686
49. Arriola C, Garg S, Anderson EJ, et al. Influenza vaccination modifies disease severity among community-dwelling adults hospitalized with influenza. Clin Infect Dis. 2017;65(8):1289-1297. doi:10.1093/cid/ cix 468

50. Talbot HK, Griffin MR, Chen Q, Zhu Y, Williams JV, Edwards KM. Effectiveness of seasonal vaccine in preventing confirmed influenza-associated hospitalizations in community dwelling older adults. $J$ Infect Dis. 2011;203(4):500-508. doi:10.1093/infdis/jiq076

51. Thompson MG, Pierse N, Sue Huang Q, et al. Influenza vaccine effectiveness in preventing influenza-associated intensive care admissions and attenuating severe disease among adults in New Zealand 2012-2015. Vaccine. 2018;36(39):5916-5925. doi:10.1016/j. vaccine.2018.07.028

52. Hsu PS, Lian IB, Chao DY. A population-based propensity score-matched study to assess the impact of repeated vaccination on vaccine effectiveness for influenza-associated hospitalization among the elderly. Clin Interv Aging. 2020;15:301-312. doi:10.2147/CIA. S238786

53. Andrew MK, MacDonald S, Godin J, et al. Persistent functional decline following hospitalization with influenza or acute respiratory illness. J Am Geriatr Soc. 2020. Online ahead of print.

54. Lees C, Godin J, McElhaney JE, et al. Frailty hinders recovery from influenza and acute respiratory illness in older adults. $J$ Infect Dis. 2020;222(3):428-437. doi:10.1093/infdis/jiaa092

55. Franceschi C, Capri M, Monti D, et al. Inflammaging and anti-inflammaging: a systemic perspective on aging and longevity emerged from studies in humans. Mech Ageing Dev. 2007;128 (1):92-105. doi:10.1016/j.mad.2006.11.016

56. Torjesen I. Covid-19: Norway investigates 23 deaths in frail elderly patients after vaccination. BMJ. 2021;372:n149. doi:10.1136/bmj. n149

57. Ruiz JB, Bell RA. Predictors of intention to vaccinate against COVID-19: results of a nationwide survey. Vaccine. 2021;39:1080-1086. doi:10.1016/j.vaccine.2021.01.010

58. Sherman SM, Smith LE, Sim J. et al. COVID-19 vaccination intention in the UK: results from the COVID-19 vaccination acceptability study (CoVAccS), a nationally representative cross-sectional survey. Hum Vaccin Immunother;2020. 1-10. doi:10.1080/ 21645515.2020.1846397

59. Alzheimer Society of Canada. COVID-19 vaccination rollout; 2021. Available from: https://alzheimer.ca/en/whats-happening/news/covid19-vaccination-rollout. Accessed February 12, 2021.

60. CanAge. Adult vaccination in Canada: cross-country report card 2021; 2021. Available from: https://www.canage.ca/vaccinereport. Accessed February 12, 2021.

61. Help Age International. Coronavirus (COVID-19). Available from: https://www.helpage.org/what-we-do/coronavirus-covid19/. Accessed February 12, 2021.
Clinical Interventions in Aging

\section{Publish your work in this journal}

Clinical Interventions in Aging is an international, peer-reviewed journal focusing on evidence-based reports on the value or lack thereof of treatments intended to prevent or delay the onset of maladaptive correlates of aging in human beings. This journal is indexed on PubMed Central, MedLine, CAS, Scopus and the Elsevier
Bibliographic databases. The manuscript management system is completely online and includes a very quick and fair peer-review system, which is all easy to use. Visit http://www.dovepress.com/ testimonials.php to read real quotes from published authors. 